\title{
Research Article \\ Solving Heat and Wave-Like Equations Using He's Polynomials
}

\section{Syed Tauseef Mohyud-Din}

Basic Sciences, HITEC University, 44000 Taxila Cantt, Pakistan

Correspondence should be addressed to Syed Tauseef Mohyud-Din, syedtauseefs@hotmail.com

Received 21 May 2009; Revised 30 June 2009; Accepted 6 July 2009

Recommended by Ji Huan He

We use He's polynomials which are calculated form homotopy perturbation method (HPM) for solving heat and wave-like equations. The proposed iterative scheme finds the solution without any discretization, linearization, or restrictive assumptions. Several examples are given to verify the reliability and efficiency of the method. The fact that suggested technique solves nonlinear problems without using Adomian's polynomials is a clear advantage of this algorithm over the decomposition method.

Copyright (C 2009 Syed Tauseef Mohyud-Din. This is an open access article distributed under the Creative Commons Attribution License, which permits unrestricted use, distribution, and reproduction in any medium, provided the original work is properly cited.

\section{Introduction}

The heat and wave-like models are the integral part of applied sciences and arise in various physical phenomena. Several techniques including spectral, characteristic, modified variational iteration and Adomian's decomposition have been used for solving these problems; see [1-3] and the references therein. Most of these techniques encounter a considerable size of difficulty. He [4-11] developed and formulated homotopy perturbation method (HPM) by merging the standard homotopy and perturbation. The homotopy perturbation method (HPM) proved to be compatible with the versatile nature of the physical problems and has been applied to a wide class of functional equations; see [1, 419] and the references therein. In this technique, the solution is given in an infinite series usually converging to an accurate solution; see [1,4-19] and the references therein. It is worth mentioning that HPM is applied without any discretization, restrictive assumption or transformation and is free from round off errors. The HPM is applied for all the nonlinear terms in the problem without discretizing either by finite difference or by spline techniques at the nodes and involves laborious calculations coupled with a strong possibility of the illconditioned resultant equations which are a complicated problem to solve. Moreover, unlike 
the method of separation of variables that requires initial and boundary conditions, the homotopy perturbation method (HPM) provides an analytical solution by using the initial conditions only. The fact that HPM solves nonlinear problems without using Adomian's polynomials is a clear advantage of this technique over the decomposition method. It is worth mentioning that $[12,13]$ introduced He's polynomials by splitting the nonlinear term and proved their compatibility with Adomian's polynomials coupled with the conclusion that He's polynomials are easier to calculate, are more user friendly, and are independent of the complexities arising in calculating the so-called Adomian's polynomials. It is to be highlighted that He's polynomials are calculated from homotopy perturbation method (HPM). Inspired and motivated by the ongoing research in this area, we use He's polynomials for solving heat and wave-like equations. It is worth mentioning that Noor and Mohyud-Din [20] introduced a homotopy approach which involves an additional term and consequently leads towards laborious and redundant calculations, whereas the approach used in Section 2 is more precise and easier to implement. Moreover, it reduces the huge unnecessary calculation arising in [20]. Several examples are given to verify the reliability and efficiency of the algorithm.

\section{Homotopy Perturbation Method and He's Polynomials}

To explain the homotopy perturbation method, we consider a general equation of the type

$$
L(u)=0,
$$

where $L$ is any integral or differential operator. We define a convex homotopy $H(u, p)$ by

$$
H(u, p)=(1-p) F(u)+p L(u)
$$

where $F(u)$ is a functional operator with known solutions $v_{0}$, which can be obtained easily. It is clear that, for

$$
H(u, p)=0,
$$

we have

$$
H(u, 0)=F(u), \quad H(u, 1)=L(u) .
$$

This shows that $H(u, p)$ continuously traces an implicitly defined curve from a starting point $H\left(v_{0}, 0\right)$ to a solution function $H(f, 1)$. The embedding parameter monotonically increases from zero to unit as the trivial problem $F(u)=0$ is continuously deforms the original problem $L(u)=0$. The embedding parameter $p \in(0,1]$ can be considered as an expanding parameter $[1,2,4-19]$. The homotopy perturbation method uses the homotopy parameter $p$ as an expanding parameter [4-11] to obtain

$$
u=\sum_{i=0}^{\infty} p^{i} u_{i}=u_{0}+p u_{1}+p^{2} u_{2}+p^{3} u_{3}+\cdots
$$


if $p \rightarrow 1$, then (2.5) corresponds to (2.2) and becomes the approximate solution of the form

$$
f=\lim _{p \rightarrow 1} u=\sum_{i=0}^{\infty} u_{i}
$$

It is well known that series (2.6) is convergent for most of the cases and also the rate of convergence is dependent on $L(u)$; see [4-11]. We assume that (2.6) has a unique solution. The comparisons of like powers of $p$ give solutions of various orders. In sum, according to $[12,13]$, He's HPM considers the solution, $u(x)$, of the homotopy equation in a series of $p$ as follows:

$$
u(x)=\sum_{i=0}^{\infty} p^{i} u_{i}=u_{0}+p u_{1}+p^{2} u_{2}+\cdots,
$$

and the method considers the nonlinear term $N(u)$ as

$$
N(u)=\sum_{i=0}^{\infty} p^{i} H_{i}=H_{0}+p H_{1}+p^{2} H_{2}+\cdots,
$$

where $H_{n}$ 's are the so-called He's polynomials $[12,13]$, which can be calculated by using the formula

$$
H_{n}\left(u_{0}, \ldots, u_{n}\right)=\frac{1}{n !} \frac{\partial^{n}}{\partial p^{n}}\left(N\left(\sum_{i=0}^{n} p^{i} u_{i}\right)\right)_{p=0}, \quad \mathrm{n}=0,1,2, \ldots
$$

\section{Numerical Applications}

In this section, we use He's polynomials which are calculated from homotopy perturbation method (HPM) for solving heat and wave-like equations.

Example $3.1([1,2])$. Consider the one-dimensional initial boundary value problem which describes the heat-like models

$$
u_{t}=\frac{1}{2} x^{2} u_{x x}, \quad 0<x<1, t>0
$$

with boundary conditions

$$
u(0, t)=0, \quad u(1, t)=e^{t},
$$

and initial conditions

$$
u(x, 0)=x^{2} .
$$


Apply the convex homotopy

$$
u_{0}+p u_{1}+p^{2} u_{2}+\cdots=x^{2}+\int_{0}^{t} \frac{1}{2} x^{2}\left(\frac{\partial^{2} u_{0}}{\partial x^{2}}+p \frac{\partial^{2} u_{1}}{\partial x^{2}}+p^{2} \frac{\partial^{2} u_{2}}{\partial x^{2}}+\cdots\right) d t
$$

Compare the coefficient of like powers of $p$

$$
\begin{aligned}
& p^{(0)}: u_{0}(x, t)=x^{2}, \\
& p^{(1)}: u_{1}(x, t)=x^{2} t, \\
& p^{(2)}: u_{2}(x, t)=x^{2} \frac{t^{2}}{2 !}, \\
& p^{(3)}: u_{3}(x, t)=x^{2} \frac{t^{3}}{3 !}, \\
& p^{(4)}: u_{4}(x, t)=x^{2} \frac{t^{4}}{4 !},
\end{aligned}
$$

where $p^{(i)} s$ are He's polynomials. The series solution is given by

$$
u(x, t)=x^{2}\left(1+t+\frac{t^{2}}{2 !}+\frac{t^{3}}{3 !}+\frac{t^{4}}{4 !}+\cdots\right)
$$

and in a closed form by

$$
u(x, t)=x^{2} e^{t}
$$

Example $3.2([1,2])$. Consider the two-dimensional initial boundary value problem which describes the heat-like models

$$
u_{t}=\frac{1}{2}\left(y^{2} u_{x x}+x^{2} u_{y y}\right), \quad 0<x, y<1, t>0
$$

with boundary conditions

$$
\begin{array}{ll}
u_{x}(0, y, t)=0, & u_{x}(1, y, t)=2 \sinh t \\
u_{y}(x, 0, t)=0, & u_{y}(x, 1, t)=2 \cosh t
\end{array}
$$

and initial conditions

$$
u(x, y, 0)=y^{2}
$$


Apply the convex homotopy method

$$
\begin{aligned}
u_{0}+p u_{1}+p^{2} u_{2}+\cdots=y^{2}+\frac{1}{2} \int_{0}^{t}( & y^{2}\left(\frac{\partial^{2} u_{0}}{\partial x^{2}}+p \frac{\partial^{2} u_{1}}{\partial x^{2}}+p^{2} \frac{\partial^{2} u_{2}}{\partial x^{2}}+\cdots\right) \\
& \left.+x^{2}\left(\frac{\partial^{2} u_{0}}{\partial y^{2}}+p \frac{\partial^{2} u_{1}}{\partial y^{2}}+p^{2} \frac{\partial^{2} u_{2}}{\partial y^{2}}+\cdots\right)\right) d s
\end{aligned}
$$

Compare the coefficient of like powers of $p$

$$
\begin{gathered}
p^{(0)}: u_{0}(x, y, t)=y^{2}, \\
p^{(1)}: u_{1}(x, y, t)=x^{2} t, \\
p^{(2)}: u_{2}(x, y, t)=y^{2} \frac{t^{2}}{2 !} \\
p^{(3)}: u_{3}(x, y, t)=x^{2} \frac{t^{3}}{3 !}, \\
p^{(4)}: u_{4}(x, y, t)=y^{2} \frac{t^{4}}{4 !},
\end{gathered}
$$

where $p^{(i)} S$ are He's polynomials. The series solution is given by

$$
u(x, y, t)=x^{2}\left(t+\frac{t^{3}}{3 !}+\frac{t^{5}}{5 !}+\cdots\right)+y^{2}\left(1+\frac{t^{2}}{2 !}+\frac{t^{4}}{4 !}+\cdots\right)
$$

and in a closed form by

$$
u(x, y, t)=x^{2} \sinh t+y^{2} \cosh t
$$

Example 3.3 ([1, 2]). Consider the three-dimensional inhomogeneous initial boundary value problem which describes the heat-like models

$$
u_{t}=x^{4} y^{4} z^{4}+\frac{1}{36}\left(x^{2} u_{x x}+y^{2} u_{y y}+z^{2} u_{z z}\right), \quad 0<x, y, z<1, t>0
$$

subject to the boundary conditions

$$
\begin{array}{ll}
u(0, y, z, t)=0, & u(1, y, z, t)=y^{4} z^{4}\left(e^{t}-1\right), \\
u(x, 0, z, t)=0, & u(x, 1, z, t)=x^{4} z^{4}\left(e^{t}-1\right), \\
u(x, y, 0, t)=0, & u(x, y, 1, t)=x^{4} y^{4}\left(e^{t}-1\right),
\end{array}
$$


and the initial conditions

$$
u(x, y, z, 0)=0
$$

Apply the convex homotopy method

$$
\begin{aligned}
& u_{0}+p u_{1}+p^{2} u_{2}+\cdots= x^{4} y^{4} z^{4} t+\frac{1}{36} p \int_{0}^{t} x^{2}\left(\frac{\partial^{2} u_{0}}{\partial x^{2}}+p \frac{\partial^{2} u_{1}}{\partial x^{2}}+p^{2} \frac{\partial^{2} u_{2}}{\partial x^{2}}+\cdots\right) d t \\
&+\frac{1}{36} p \int_{0}^{t}\left(y^{2}\left(\frac{\partial^{2} u_{0}}{\partial y^{2}}+p \frac{\partial^{2} u_{1}}{\partial y^{2}}+p^{2} \frac{\partial^{2} u_{2}}{\partial y^{2}}+\cdots\right)\right. \\
&\left.+z^{2}\left(\frac{\partial^{2} u_{0}}{\partial z^{2}}+p \frac{\partial^{2} u_{1}}{\partial z^{2}}+\cdots\right)\right) d t
\end{aligned}
$$

Compare the coefficient of like powers of $p$

$$
\begin{aligned}
& p^{(0)}: u_{0}(x, y, z, t)=x^{4} y^{4} z^{4} t \\
& p^{(1)}: u_{1}(x, y, z, t)=x^{4} y^{4} z^{4} \frac{t^{2}}{2 !} \\
& p^{(2)}: u_{2}(x, y, z, t)=x^{4} y^{4} z^{4} \frac{t^{3}}{3 !} \\
& p^{(3)}: u_{3}(x, y, z, t)=x^{4} y^{4} z^{4} \frac{t^{4}}{4 !}
\end{aligned}
$$

where $p^{(i)} s$ are He's polynomials. The series solution is given by

$$
u(x, y, t)=x^{4} y^{4} z^{4}\left(t+\frac{t^{2}}{2 !}+\frac{t^{3}}{3 !}+\frac{t^{4}}{4 !}+\cdots\right)
$$

and in a closed form by

$$
u(x, y, z, t)=x^{4} y^{4} z^{4}\left(e^{t}-1\right)
$$

Example 3.4. Consider the following nonlinear heat-like model:

$$
u_{t}=u_{x x}+\frac{k}{x} u_{x}-(2+2 k) u-4 u \ln (u)
$$


subject to the initial conditions

$$
u(0, t)=\exp (\exp (-4 t))
$$

Apply the convex homotopy method

$$
\begin{aligned}
u_{0}+p u_{1}+p^{2} u_{2}+\cdots \\
=\exp (\exp (-4 t))+p \int_{0}^{t}\left(\frac{\partial^{2} u_{0}}{\partial x^{2}}+p \frac{\partial^{2} u_{1}}{\partial x^{2}}+p^{2} \frac{\partial^{2} u_{2}}{\partial x^{2}}+\cdots\right) d t \\
\quad+p \int_{0}^{t}\left(\frac{k}{x}\left(\frac{\partial u_{0}}{\partial x}+p \frac{\partial u_{1}}{\partial x}+p^{2} \frac{\partial u_{2}}{\partial x}+\cdots\right)-(2+2 k)\left(u_{0}+p u_{1}+p^{2} u_{2}+\cdots\right)\right) d t \\
\quad-4 p \int_{0}^{t}\left(u_{0}+p u_{1}+p^{2} u_{2}+\cdots\right)\left(\ln u_{0}+p \ln u_{1}+\cdots\right) d t
\end{aligned}
$$

Compare the coefficient of like powers of $p$

$$
\begin{aligned}
& p^{(0)}: u_{0}(x, t)=e^{e^{-4 t}}, \\
& p^{(1)}: u_{1}(x, t)=\frac{x^{2}}{1 !} e^{e^{-4 t}}, \\
& p^{(2)}: u_{2}(x, t)=\frac{x^{4}}{2 !} e^{e^{-4 t}}, \\
& p^{(3)}: u_{3}(x, t)=\frac{x^{6}}{3 !} e^{e^{-4 t}}, \\
& p^{(4)}: u_{4}(x, t)=\frac{x^{8}}{4 !} e^{e^{-4 t}}, \\
& p^{(5)}: u_{2}(x, t)=\frac{x^{10}}{5 !} e^{e^{-4 t}},
\end{aligned}
$$

where $p^{(i)} s$ are He's polynomials. The series solution is given by

$$
u(x, t)=\left(1+\frac{x^{2}}{1 !}+\frac{x^{4}}{2 !}+\frac{x^{6}}{3 !}+\frac{x^{8}}{4 !}+\frac{x^{10}}{5 !}+\cdots\right) e^{e^{-4 t}}
$$

and the closed form solution is given as

$$
u(x, t)=e^{x^{2}+e^{e^{-4 t}}}
$$


Example $3.5([1,2])$. Consider the one-dimensional initial boundary value problem which describes the wave-like models

$$
u_{t t}=\frac{1}{2} x^{2} u_{x x}, \quad 0<x<1, t>0
$$

subject to the boundary conditions

$$
u(x, t)=x, \quad u(1, t)=1+\sinh t
$$

and initial conditions

$$
u(x, 0)=x, \quad u_{t}(x, 0)=x^{2} .
$$

Apply the convex homotopy method

$$
u_{0}+p u_{1}+p^{2} u_{2}+\cdots=x^{2}+\frac{1}{2} \iint_{0}^{t} x^{2}\left(\frac{\partial^{2} u_{0}}{\partial x^{2}}+p \frac{\partial^{2} u_{1}}{\partial x^{2}}+p^{2} \frac{\partial^{2} u_{2}}{\partial x^{2}}+\cdots\right) d t d t
$$

Compare the coefficient of like powers of $p$

$$
\begin{aligned}
& p^{(0)}: u_{0}(x, t)=x+x^{2} t \\
& p^{(1)}: u_{1}(x, t)=x^{2} \frac{t^{3}}{3 !} \\
& p^{(2)}: u_{2}(x, t)=x^{2} \frac{t^{5}}{5 !} \\
& p^{(3)}: u_{3}(x, t)=x^{2} \frac{t^{7}}{7 !}
\end{aligned}
$$

where $p^{(i)} s$ are He's polynomials. The series solution is given by

$$
u(x, t)=x+x^{2}\left(t+\frac{t^{3}}{3 !}+\frac{t^{5}}{5 !}+\frac{t^{7}}{7 !}+\ldots\right)
$$

and in a closed form by

$$
u(x, t)=x+x^{2} \sinh t
$$


Example $3.6([1,2])$. Consider the two-dimensional initial boundary value problem which describes the wave-like models

$$
u_{t t}=\frac{1}{12}\left(x^{2} u_{x x}+y^{2} y_{y y}\right), \quad 0<x, y<1, t>0,
$$

subject to the Neumann boundary conditions

$$
\begin{array}{ll}
u_{x}(0, y, t)=0, & u_{x}(1, y, t)=4 \cosh t \\
u_{y}(x, 0, t)=0, & u_{y}(x, 1, t)=4 \sinh t
\end{array}
$$

and initial conditions

$$
u(x, y, 0)=x^{4}, \quad u_{t}(x, y, 0)=y^{4} .
$$

Apply the convex homotopy method

$$
\begin{aligned}
u_{0}+p u_{1}+p^{2} u_{2}+\cdots= & \left(x^{4}+y^{4} t\right)+\frac{1}{12} \iint_{0}^{t} x^{2}\left(\frac{\partial^{2} u_{0}}{\partial x^{2}}+p \frac{\partial^{2} u_{1}}{\partial x^{2}}+p^{2} \frac{\partial^{2} u_{2}}{\partial x^{2}}+\cdots\right) d t d t \\
& +\frac{1}{12} \iint_{0}^{t} y^{2}\left(\frac{\partial^{2} u_{0}}{\partial y^{2}}+p \frac{\partial^{2} u_{1}}{\partial y^{2}}+p^{2} \frac{\partial^{2} u_{2}}{\partial y^{2}}+\cdots\right) d t d t
\end{aligned}
$$

Compare the coefficient of like powers of $p$

$$
\begin{gathered}
p^{(0)}: u_{0}(x, y, t)=x^{4}+y^{4} t, \\
p^{(1)}: u_{1}(x, y, t)=x^{4} \frac{t^{2}}{2 !}+y^{4} \frac{t^{3}}{3 !}, \\
p^{(2)}: u_{2}(x, y, t)=x^{4} \frac{t^{4}}{4 !}+y^{4} \frac{t^{5}}{5 !}, \\
p^{(3)}: u_{3}(x, y, t)=x^{4} \frac{t^{6}}{6 !}+y^{4} \frac{t^{7}}{7 !},
\end{gathered}
$$

where $p^{(i)} s$ are He's polynomials. The series solution is given by

$$
u(x, y, t)=x^{4}\left(1+\frac{t^{2}}{2 !}+\frac{t^{4}}{4 !}+\cdots\right)+y^{4}\left(1+\frac{t^{3}}{3 !}+\frac{t^{5}}{5 !}+\cdots\right)
$$

and in a closed form by

$$
u(x, y, t)=x^{4} \cosh t+y^{4} \sinh t
$$


Example $3.7([1,2])$. Consider the three-dimensional inhomogeneous initial boundary value problem which describes the wave-like models

$$
u_{t t}=\left(x^{2}+y^{2}+z^{2}\right)+\frac{1}{2}\left(x^{2} u_{x x}+y^{2} u_{y y}+z^{2} u_{z z}\right), \quad 0<x, y, z<1, t>0
$$

subject to the boundary conditions

$$
\begin{aligned}
& u(0, y, z, t)=y^{2}\left(e^{t}-1\right)+z^{2}\left(e^{-t}-1\right), \quad u(1, y, z, t)=\left(1+y^{2}\right)\left(e^{t}-1\right)+z^{2}\left(e^{-t}-1\right), \\
& u(x, 0, z, t)=x^{2}\left(e^{t}-1\right)+z^{2}\left(e^{-t}-1\right), \quad u(x, 1, z, t)=\left(1+x^{2}\right)\left(e^{t}-1\right)+z^{2}\left(e^{-t}-1\right), \\
& u(x, y, 0, t)=\left(x^{2}+y^{2}\right)\left(e^{t}-1\right), \quad u(x, y, 1, t)=\left(x^{2}+y^{2}\right)\left(e^{t}-1\right)+\left(e^{-t}-1\right),
\end{aligned}
$$

and the initial conditions

$$
u(x, y, z, 0)=0, \quad u_{t}(x, y, z, 0)=x^{2}+y^{2}-z^{2}
$$

Apply the convex homotopy method

$$
\begin{gathered}
u_{0}+p u_{1}+p^{2} u_{2}+\cdots=\left(x^{2}+y^{2}-z^{2}\right) t+\frac{1}{2} \iint_{0}^{t} x^{2}\left(\frac{\partial^{2} u_{0}}{\partial x^{2}}+p \frac{\partial^{2} u_{1}}{\partial x^{2}}+p^{2} \frac{\partial^{2} u_{2}}{\partial x^{2}}+\cdots\right) d t d t \\
+\frac{1}{2} \iint_{0}^{t}\left(y^{2}\left(\frac{\partial^{2} u_{0}}{\partial y^{2}}+p \frac{\partial^{2} u_{1}}{\partial y^{2}}+p^{2} \frac{\partial^{2} u_{2}}{\partial y^{2}}+\cdots\right)\right. \\
\left.+z^{2}\left(\frac{\partial^{2} u_{0}}{\partial z^{2}}+p \frac{\partial^{2} u_{1}}{\partial z^{2}}+p^{2} \frac{\partial^{2} u_{2}}{\partial z^{2}}+\cdots\right)\right) d t d t
\end{gathered}
$$

Compare the coefficient of like powers of $p$ and proceede as before, the series solution is given by

$$
u(x, y, t)=\left(x^{2}+y^{2}\right)\left(t+\frac{t^{2}}{2 !}+\frac{t^{3}}{3 !}+\frac{t^{4}}{4 !}+\cdots\right)+z^{2}\left(-t+\frac{t^{2}}{2 !}+\frac{t^{3}}{3 !}+\frac{t^{4}}{4 !}+\cdots\right)
$$

and in a closed form by

$$
u(x, y, z, t)=\left(x^{2}+y^{2}\right) e^{t}+z^{2} e^{-t}-\left(x^{2}+y^{2}+z^{2}\right) .
$$




\section{Conclusion}

In this paper, we use He's polynomials which are calculated from homotopy perturbation method (HPM) for solving heat and wave-like equations. The method is applied in a direct way without using linearization, transformation, discretization, or restrictive assumptions. It may be concluded that the proposed scheme is very powerful and efficient in finding the analytical solutions for a wide class of boundary value problems. The method gives more realistic series solutions that converge very rapidly in physical problems. It is also observed that He's polynomials are compatible with Adomian's Polynomials but are easier to calculate and are more user friendly.

\section{Acknowledgments}

The author is highly grateful to the referees and Professor Dr. Ji-Huan He for their very constructive comments. The author would like to thank Brig (R) Qamar Zaman, Vice Chancellor, HITEC University, Taxila Cantt, Pakistan for the provision of conducive environs for research.

\section{References}

[1] M. A. Noor and S. T. Mohyud-Din, "Modified variational iteration method for heat and wave-like equations," Acta Applicandae Mathematicae, vol. 104, no. 3, pp. 257-269, 2008.

[2] A.-M. Wazwaz and A. Gorguis, "Exact solutions for heat-like and wave-like equations with variable coefficients," Applied Mathematics and Computation, vol. 149, no. 1, pp. 15-29, 2004.

[3] R. Wilcox, "Closed-form solution of the differential equation $\left(\partial^{2} / \partial x \partial y+a x(\partial / \partial x)+b y(\partial / \partial y)+c x y+\right.$ $\partial / \partial t) P(x, y, t)=0$ by normal-ordering exponential operators," Journal of Mathematical Physics, vol. 11, no. 4, pp. $1235-1237,1970$.

[4] J.-H. He, "An elementary introduction to recently developed asymptotic methods and nanomechanics in textile engineering," International Journal of Modern Physics B, vol. 22, no. 21, pp. 3487-3578, 2008.

[5] J.-H. He, "Recent development of the homotopy perturbation method," Topological Methods in Nonlinear Analysis, vol. 31, no. 2, pp. 205-209, 2008.

[6] J.-H. He, "Some asymptotic methods for strongly nonlinear equations," International Journal of Modern Physics B, vol. 20, no. 10, pp. 1141-1199, 2006.

[7] J.-H. He, "Homotopy perturbation method for solving boundary value problems," Physics Letters A, vol. 350, no. $1-2$, pp. 87-88, 2006.

[8] J.-H. He, "Comparison of homotopy perturbation method and homotopy analysis method," Applied Mathematics and Computation, vol. 156, no. 2, pp. 527-539, 2004.

[9] J.-H. He, "Homotopy perturbation method for bifurcation of nonlinear problems," International Journal of Nonlinear Sciences and Numerical Simulation, vol. 6, no. 2, pp. 207-208, 2005.

[10] J.-H. He, "The homotopy perturbation method for nonlinear oscillators with discontinuities," Applied Mathematics and Computation, vol. 151, no. 1, pp. 287-292, 2004.

[11] J.-H. He, "A coupling method of a homotopy technique and a perturbation technique for non-linear problems," International Journal of Non-Linear Mechanics, vol. 35, no. 1, pp. 37-43, 2000.

[12] A. Ghorbani and J. Saberi-Nadjafi, "He's homotopy perturbation method for calculating adomian polynomials," International Journal of Nonlinear Sciences and Numerical Simulation, vol. 8, no. 2, pp. 229-232, 2007.

[13] A. Ghorbani, "Beyond Adomian polynomials: He polynomials," Chaos, Solitons E Fractals, vol. 39, no. 3, pp. 1486-1492, 2009.

[14] S. T. Mohyud-Din, M. A. Noor, and K. I. Noor, "Traveling wave solutions of seventh-order generalized $\mathrm{KdV}$ equations using He's polynomials," International Journal of Nonlinear Sciences and Numerical Simulation, vol. 10, no. 2, pp. 227-233, 2009.

[15] S. T. Mohyud-Din and M. A. Noor, "Homotopy perturbation method for solving fourth-order boundary value problems," Mathematical Problems in Engineering, vol. 2007, Article ID 98602, 15 pages, 2007. 
[16] S. T. Mohyud-Din, M. A. Noor, and K. I. Noor, "Homotopy perturbation method for unsteady flow of gas through a porous medium," International Journal of Modern Physics B. In press.

[17] S. T. Mohyud-Din, M. A. Noor, and K. I. Noor, "On the coupling of polynomials with correction functional," International Journal of Modern Physics B. In press.

[18] M. A. Noor and S. T. Mohyud-Din, "Homotopy perturbation method for solving nonlinear higherorder boundary value problems," International Journal of Nonlinear Sciences and Numerical Simulation, vol. 9, no. 4, pp. 395-408, 2008.

[19] L. Xu, "He's homotopy perturbation method for a boundary layer equation in unbounded domain," Computers \& Mathematics with Applications, vol. 54, no. 7-8, pp. 1067-1070, 2007.

[20] M. A. Noor and S. T. Mohyud-Din, "Modified variational iteration method for heat and wave-like equations," Acta Applicandae Mathematicae, vol. 104, no. 3, pp. 257-269, 2008. 


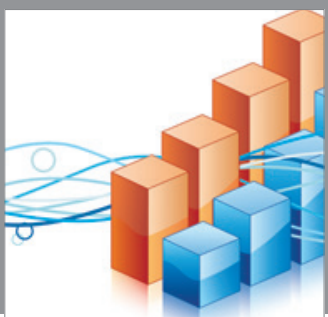

Advances in

Operations Research

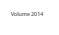

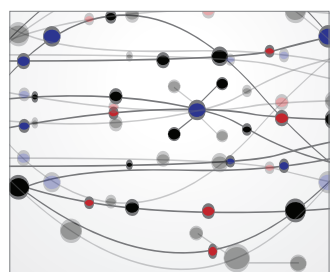

\section{The Scientific} World Journal
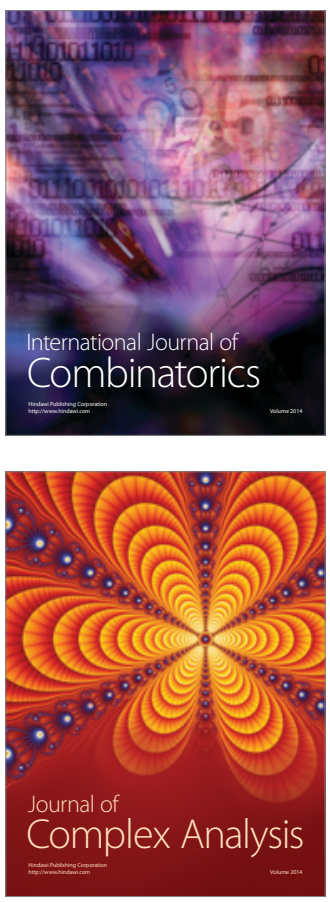

International Journal of

Mathematics and

Mathematical

Sciences
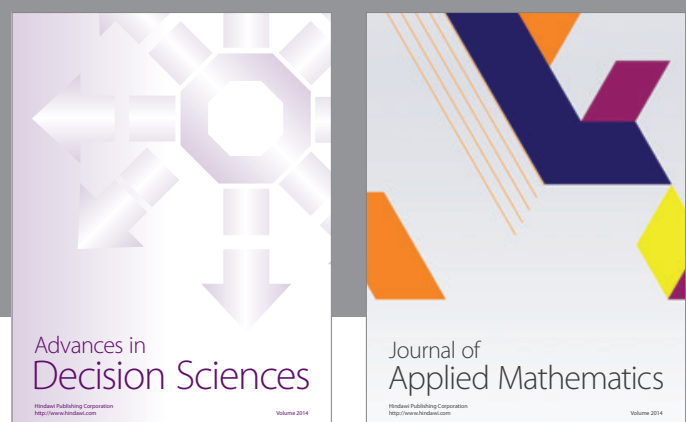

Journal of

Applied Mathematics
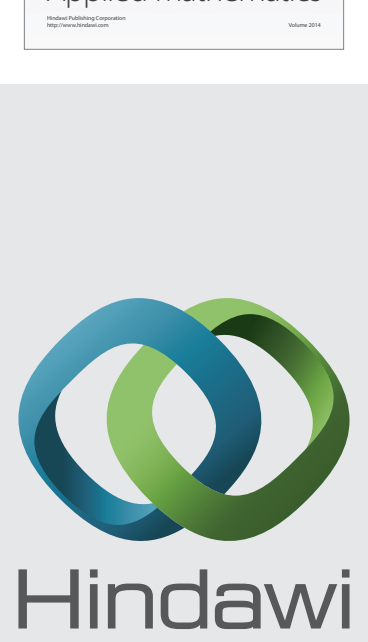

Submit your manuscripts at http://www.hindawi.com
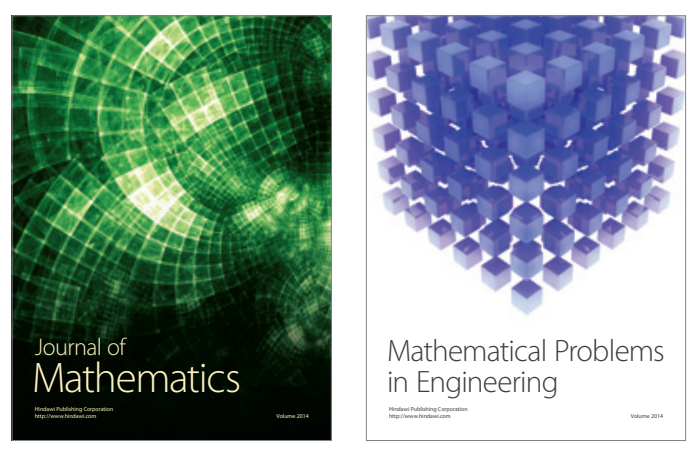

Mathematical Problems in Engineering
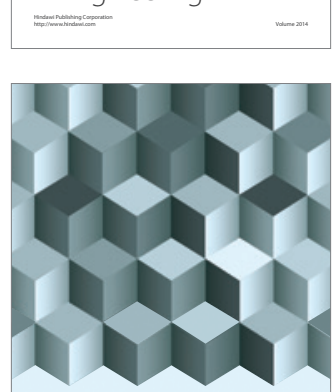

Journal of

Function Spaces
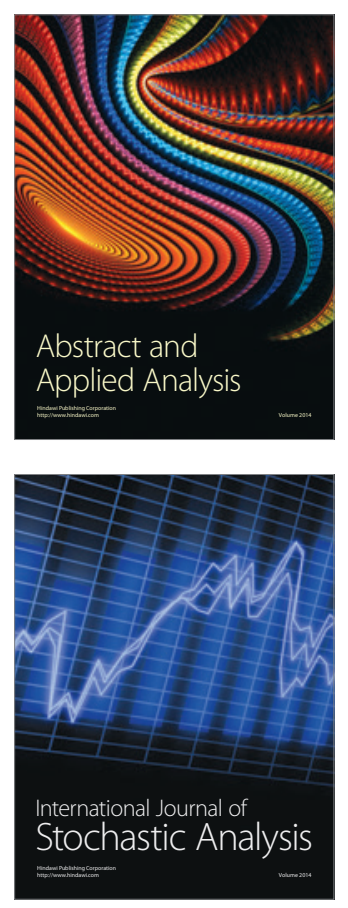

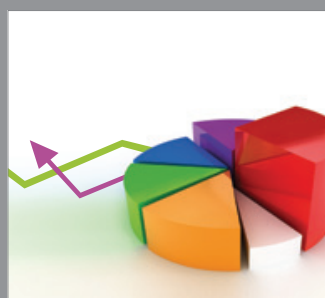

ournal of

Probability and Statistics

Promensencen
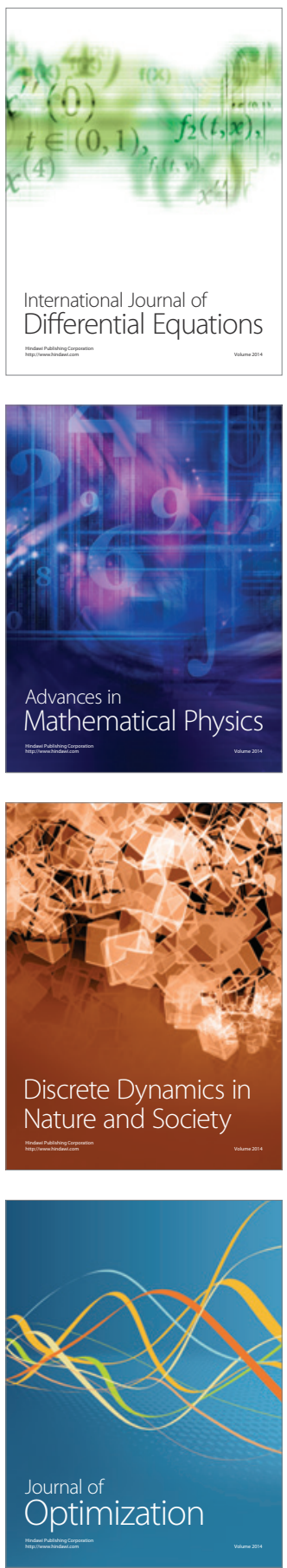